\title{
Correlated tunneling and the instability of the fractional quantum Hall edge
}

\author{
Dror Orgad and Oded Agam \\ The Racah Institute of Physics, The Hebrew University, Jerusalem, 91904, Israel
}

(Dated: October 28, 2018)

\begin{abstract}
We consider a class of interaction terms that describes correlated tunneling of composite fermions between effective Landau levels. Despite being generic and of similar strength to that of the usual density-density couplings, these terms are not included in the accepted theory of the edges of fractional quantum Hall systems. Here we show that they may lead to an instability of the edge towards a new reconstructed state with additional channels, and thereby demonstrate the incompleteness of the traditional edge theory.

PACS numbers: 71.10.Pm
\end{abstract}

Much of our theoretical understanding of the edge physics in the fractional quantum Hall (FQH) regime is based on the chiral Tomonaga-Luttinger (CTL) model put forward by Wen [1]. For the Jain fractions $\nu=$ $n /(2 n \pm 1)$, this low-energy effective theory may be derived by considering small fluctuations around the meanfield configuration which describes the bulk of the sample as $n$ filled Landau-levels of composite fermions [1, 2, 3]. The theory associates a chiral channel with each of these levels where it crosses the Fermi energy near the edge, and includes the electronic correlations of the bulk FQH liquid through a non-trivial exchange statistics between the excitations of the different channels.

A central prediction of this edge model is a non-linear tunneling conductance $I \sim V^{\alpha}$, with an exponent $\alpha=3$ over a range of filling factors $1 / 3<\nu<1 / 2$. While tunneling experiments $[4,5,6,6]$ have indeed found a powerlaw characteristic, the exponent scales as $\alpha \approx a / \nu+b$, where $a$ and $b$ are non-universal constants, in contradiction to the predicted value [8]. This discrepancy suggests that the accepted CTL theory does not incorporate some important elements for the understanding of the FQH edge physics. In this Letter we concentrate on one such element, namely a class of interaction terms describing correlated tunneling of composite fermions between edge states, and show that it may lead to edge reconstruction, where the new structure supports additional channels.

In order to clarify the origin of such processes, consider the interaction energy between the electrons written in terms of composite fermions. To this end we transform from the original electronic operator $\Psi(\mathbf{r})$ to a composite fermion operator $\psi(\mathbf{r})=e^{i \Lambda(\mathbf{r})} \Psi(\mathbf{r})$, via a Chern-Simons phase $\Lambda(\mathbf{r})$, and expand $\psi(\mathbf{r})$ using the composite fermion annihilation operators on the $n$ occupied effective Landau levels $\psi(\mathbf{r})=\sum_{i=1}^{n} \psi_{i}(\mathbf{r})$ [9]. The result is

$$
\begin{aligned}
& \frac{1}{2} \int d^{2} r d^{2} r^{\prime} \rho_{e}(\mathbf{r}) V\left(\mathbf{r}-\mathbf{r}^{\prime}\right) \rho_{e}\left(\mathbf{r}^{\prime}\right) \\
& =\frac{1}{2} \sum_{i j k l=1}^{n} \int d^{2} r d^{2} r^{\prime} \psi_{i}^{\dagger}(\mathbf{r}) \psi_{j}(\mathbf{r}) V\left(\mathbf{r}-\mathbf{r}^{\prime}\right) \psi_{k}^{\dagger}\left(\mathbf{r}^{\prime}\right) \psi_{l}\left(\mathbf{r}^{\prime}\right),
\end{aligned}
$$

where $\rho_{e}=\Psi^{\dagger} \Psi$. In the traditional CTL model only the
Hartree terms $i=j, k=l$ are kept. When the interaction potential is short ranged the Fock combination $i=l$, $j=k$, gives a similar contribution but with an opposite sign and may be incorporated via a renormalization of the density-density coupling. Our focus lies with the effect of additional terms with $i=j$ but $k \neq l$ (or vice versa) in (11). These processes, which we dub correlated tunneling, correspond to tunneling of composite fermions between different Landau levels with an amplitude that depends on the total density. Correlated tunneling is of the same order as the Hartree-Fock interactions [10] when the interaction strength is comparable to the gap between the effective Landau levels. This condition, which is typically satisfied in the FQH regime, ensures that correlated tunneling conserves both energy and momentum.

The possibility of edge reconstruction due to the competition between a long-range Hartree term and a shortrange exchange interaction, has been realized in the context of the integer quantum Hall effect [11]. Composite fermions Hartree-Fock calculations have found a similar transition, triggered by a softening of the confining potential, in a $\nu=1 / 3$ edge (but not at higher fillings) 12]. Edge reconstruction was also detected in exact diagonalization studies of FQH systems with sharp edges [13, 14, 15], and was attributed to the finite range of the interactions [16]. Here we offer a new avenue for the instability via the correlated tunneling processes.

A qualitative picture for the nature of this instability follows from a toy model where two states, occupied by identical charged bosons, are coupled by correlated tunneling. Let $N_{1}$ and $N_{2}$ represent the number of particles in each one of these states, and $H_{C}=$ $\left(N_{1}+N_{2}-N_{T}\right)^{2} / 2 C$ be the charging energy of the system, where $C$ plays the role of capacitance, and $N_{T}$ is a constant fixed by the chemical potential. The correlated tunneling is represented by a term of the form $H_{C T}=\lambda\left(N_{1}+N_{2}\right)\left(b_{1}^{\dagger} b_{2}+b_{2}^{\dagger} b_{1}\right)$, where $b_{j}^{\dagger}$ denotes a creation operators of a particle in state $j$, and $\lambda$ is a constant which characterizes the strength of the process. The total Hamiltonian of the system is $H=H_{C}+H_{C T}$, and for $\lambda=0$ its ground state is set by the condition $N_{1}+N_{2}=N_{T}$. For $\lambda \neq 0$ the problem can be simplified 
by the transformation $b_{ \pm}=\left(b_{1} \pm b_{2}\right) / \sqrt{2}$, which brings the Hamiltonian to the form

$$
H=\sum_{\alpha, \beta= \pm} A_{\alpha \beta} N_{\alpha} N_{\beta}-\frac{N_{T}}{C}\left(N_{+}+N_{-}\right)+\frac{N_{T}^{2}}{2 C}
$$

where $N_{ \pm}=b_{ \pm}^{\dagger} b_{ \pm}$, and

$$
A=\frac{1}{2 C}\left(\begin{array}{cc}
1+2 C \lambda & 1 \\
1 & 1-2 C \lambda
\end{array}\right) .
$$

From Eq. (2) it is evident that the eigenvalues of the matrix $A$ determine the nature of the ground state. For any finite $\lambda$, one of these eigenvalues, $\left(1 \pm \sqrt{1+4 \lambda^{2} C^{2}}\right) / 2 C$, is negative, and the system becomes unstable. This instability drives $N_{-} \rightarrow \pm \infty$ while $N_{+} \approx(1-\lambda C) N_{T}-$ $(1-2 \lambda C) N_{-}$for $\lambda C \ll 1$. Thus, unlike the case $\lambda=0$ where the minimum energy occurs for zero charging, correlated tunneling leads to a divergence of the total charge $N_{1}+N_{2}-N_{T} \simeq C \lambda\left(2 N_{-}-N_{T}\right) \rightarrow \pm \infty$. This is because depleting or filling up the system with particles, reduces the kinetic energy associated with the tunneling.

This simple consideration points to the possibility that correlated tunneling may also play an important role in determining the nature of the ground state of the FQH edge. As a test case for such a scenario we focus our attention on the $\nu=2 / 5$ edge and take as our starting point the CTL model whose action in imaginary time is

$$
\begin{aligned}
S_{0} & =\frac{1}{4 \pi} \int d x d \tau \sum_{i, j=1}^{2}\left(i \partial_{x} \phi_{i} K_{i j}^{-1} \partial_{\tau} \phi_{j}+\partial_{x} \phi_{i} V_{i j} \partial_{x} \phi_{j}\right) \\
& +\frac{\pi}{L} \int d \tau \sum_{i, j=1}^{2} N_{i} V_{i j} N_{j} .
\end{aligned}
$$

By inverting the Chern-Simons transformation, the electronic operator may be expanded according to $\Psi(\mathbf{r})=$ $e^{-i \Lambda(\mathbf{r})} \sum_{i=1}^{n} \psi_{i}(\mathbf{r}) \equiv \sum_{i=1}^{n} \Psi_{i}(\mathbf{r})$. Each bosonic field $\phi_{i}$ is related to the projection of $\Psi_{i}(\mathbf{r})$ on the gapless edge modes [3]. Denoting the projections by $\Psi_{i}(x)$ one finds

$$
\Psi_{i}(x)=\frac{F_{i}}{\sqrt{2 \pi a}} e^{\frac{2 \pi i}{L} N_{i} x+i \phi_{i}(x)},
$$

with corresponding normal ordered densities

$$
: \rho_{i}(x):=: \Psi_{i}(x)^{\dagger} \Psi_{i}(x):=\sum_{j=1}^{m} K_{i j}^{-1}\left(\frac{1}{2 \pi} \partial_{x} \phi_{j}+\frac{1}{L} N_{i}\right) .
$$

Here $L$ is the length of the edge and $a$ is a short distance cutoff of the order of the magnetic length. The Klein factors, $F_{i}$, and the conjugated number operators, $N_{i}$, obey the algebra $\left\{F_{i}, F_{j}\right\}=0$ for $i \neq j,\left\{F_{i}, F_{j}^{\dagger}\right\}=2 \delta_{i j}$ and $\left[F_{i}, N_{j}\right]=F_{i} \delta_{i j}$. Finally, the statistics of the edge channels and their interactions are given by the matrices

$$
K=\left(\begin{array}{ll}
3 & 2 \\
2 & 3
\end{array}\right), \quad V=\left(\begin{array}{cc}
v & g \\
g & v
\end{array}\right) .
$$

The matrix $V$ contains the strength $g$ of the short-range interaction between the fermions while $v$ is the velocity of the channels as determined by the confining potential and the interactions. For simplicity we have assumed the same velocity for the two channels and ignored the difference in their Fermi wavevectors which become irrelevant in the strong interaction regime which we consider.

We derive an effective one-dimensional action describing the correlated tunneling processes by integrating Eq. (11) over the coordinate perpendicular to the edge and replacing the composite fermion operators by their projections $\psi_{i}(x)$ on the edge modes. Taking $\psi_{i}^{\dagger}(x) \psi_{j}(x)=$ $\Psi_{i}^{\dagger}(x) \Psi_{j}(x)$ [17], and using Eq. (5), we find

$$
\begin{aligned}
S_{1} & =\int d x d \tau\left(\bar{\lambda}+\lambda \sum_{i=1}^{2}: \psi_{i}^{\dagger} \psi_{i}:\right)\left(\psi_{1}^{\dagger} \psi_{2}+\text { h.c. }\right) \\
& =\frac{1}{4 \pi a} \int d x d \tau\left[F_{1}^{\dagger} F_{2} e^{\frac{2 \pi i}{L}\left(N_{2}-N_{1}\right) x+i \phi_{2}-i \phi_{1}}+\text { h.c. }\right] \\
& \times\left[2 \bar{\lambda}+\frac{\lambda}{5 \pi}\left(\partial_{x} \phi_{1}+\partial_{x} \phi_{2}+\frac{2 \pi}{L}\left(N_{1}+N_{2}\right)\right)\right],
\end{aligned}
$$

where we have used that $\sum_{i=1}^{n} K_{i j}^{-1}=\nu / n$ for a Jain fraction $\nu=n /(2 n \pm 1)[3]$. The strength of the correlated tunneling is characterized by the coupling constant $\lambda$ and we have also included a term, proportional to $\bar{\lambda}$, describing simple tunneling between the levels, whose origin is the constant average value of the density in Eq. (11).

The action $S=S_{0}+S_{1}$ can be diagonalized exactly. To this end we add to it the action of a free chiral auxiliary field, $\phi_{0}$, with velocity $v_{0}=v-g$ [18]

$$
\begin{aligned}
S_{\text {aux }} & =\frac{1}{4 \pi} \int d x d \tau\left[i \partial_{x} \phi_{0} \partial_{\tau} \phi_{0}+v_{0}\left(\partial_{x} \phi_{0}\right)^{2}\right] \\
& +\frac{\pi v_{0}}{L} \int d \tau N_{0}^{2},
\end{aligned}
$$

and transform to new fields, $\varphi_{i}=\sum_{j} A_{i j} \phi_{j}$, and number operators $\mathcal{N}_{i}=\sum_{j} B_{i j} N_{j}$ [19], with

$$
A=\left(\begin{array}{ccc}
\frac{1}{\sqrt{2}} & \frac{1}{2} & -\frac{1}{2} \\
\frac{1}{\sqrt{2}} & -\frac{1}{2} & \frac{1}{2} \\
0 & \frac{1}{\sqrt{10}} & \frac{1}{\sqrt{10}}
\end{array}\right), \quad B=\frac{1}{2}\left(\begin{array}{ccc}
1 & 1 & -1 \\
1 & -1 & 1 \\
-1 & 1 & 1
\end{array}\right) \text {. }
$$

Defining new Klein factors $\mathcal{F}_{0}=F_{0} F_{1}, \mathcal{F}_{1}=F_{0} F_{2}$ and $\mathcal{F}_{2}=F_{1} F_{2}$, which are readily verified to obey the required commutation algebra among themselves and with the $\mathcal{N}_{i}$, allows us to introduce the fermions

$$
\xi_{i}(x)=\frac{\mathcal{F}_{i}}{\sqrt{2 \pi a}} e^{\frac{2 \pi i}{L} \mathcal{N}_{i} x+i \varphi_{i}(x)}, \quad i=0,1
$$

in terms of which the action $S=S_{0}+S_{1}+S_{\text {aux }}$ reads

$$
S=\int d x d \tau \sum_{j=0,1}\left(\xi_{j}^{\dagger} \partial_{\tau} \xi_{j}-: i v_{n} \xi_{j}^{\dagger} \partial_{x} \xi_{j}:\right)
$$




$$
\begin{aligned}
& +\frac{1}{4 \pi} \int d x d \tau\left[i \partial_{x} \varphi_{2} \partial_{\tau} \varphi_{2}+v_{c}\left(\partial_{x} \varphi_{2}\right)^{2}\right] \\
& +\frac{\pi}{L} \int d \tau\left[\frac{v_{n}}{2}\left(\mathcal{N}_{0}+\mathcal{N}_{1}\right)^{2}+v_{c} \mathcal{N}_{c}^{2}\right] \\
& +\int d x d \tau\left[\bar{\lambda}+\frac{\lambda}{\sqrt{10} \pi}\left(\partial_{x} \varphi_{2}+\frac{2 \pi}{L} \mathcal{N}_{c}\right)\right] \\
& \times\left(\xi_{0}^{\dagger} \xi_{1}+\xi_{1}^{\dagger} \xi_{0}\right),
\end{aligned}
$$

where $\mathcal{N}_{c}=\left(\mathcal{N}_{0}+\mathcal{N}_{1}+2 \mathcal{N}_{2}\right) / \sqrt{10}=\left(N_{1}+N_{2}\right) / \sqrt{10}$. The fields $\xi_{0,1}$ which involve the neutral edge mode $\phi_{1}-\phi_{2}$ move with its velocity $v_{n}=v-g$, while $\varphi_{2}$ which creates the symmetric combination $\phi_{1}+\phi_{2}$ propagates with the velocity of the charged edge mode $v_{c}=5(v+g)$.

Next, we transform to the even and odd combinations of $\xi_{0,1}$ and bosonize once again

$$
\xi_{ \pm}=\frac{1}{\sqrt{2}} e^{ \pm i \frac{\bar{\lambda}}{v_{n}} x}\left(\xi_{0} \pm \xi_{1}\right)=\frac{\mathcal{F}_{ \pm}}{\sqrt{2 \pi a}} e^{\frac{2 \pi i}{L} \mathcal{N}_{ \pm} x+i \varphi_{ \pm}(x)} .
$$

This step eliminates $\bar{\lambda}$ from $S$ and turns the correlated tunneling term into a density-density interaction between $\varphi_{2}$ and $\varphi_{ \pm}$. Finally, the resulting quadratic action can be diagonalized by the transformation

$$
\left(\begin{array}{c}
\theta_{0}, Q_{0} \\
\theta_{1}, Q_{1} \\
\theta_{2}, Q_{2}
\end{array}\right)=\left(\begin{array}{ccc}
\frac{1}{\sqrt{2}} & \frac{1}{\sqrt{2}} & 0 \\
\frac{\cos \gamma}{\sqrt{2}} & -\frac{\cos \gamma}{\sqrt{2}} & -\sin \gamma \\
\frac{\sin \gamma}{\sqrt{2}} & -\frac{\sin \gamma}{\sqrt{2}} & \cos \gamma
\end{array}\right)\left(\begin{array}{c}
\varphi_{+}, \mathcal{N}_{+} \\
\varphi_{-}, \mathcal{N}_{-} \\
\varphi_{2}, \mathcal{N}_{c}
\end{array}\right)
$$

where the rotation angle

$$
\tan \gamma=\sqrt{\left(v_{n}-u_{1}\right) /\left(u_{2}-v_{n}\right)},
$$

is determined by the velocities

$$
u_{1,2}=\frac{1}{2}\left[v_{c}+v_{n} \mp \sqrt{\left(v_{c}-v_{n}\right)^{2}+4 \lambda^{2} / 5 \pi^{2}}\right],
$$

of the physical modes in the diagonalized action

$$
\begin{aligned}
S & =\frac{1}{4 \pi} \int d x d \tau \sum_{j=0}^{2}\left[i \partial_{x} \theta_{j} \partial_{\tau} \theta_{j}+u_{j}\left(\partial_{x} \theta_{j}\right)^{2}\right] \\
& +\frac{\pi}{L} \int d \tau\left(\sum_{j=0}^{2} u_{j} Q_{j}^{2}+u_{0} Q_{0}^{2}\right) .
\end{aligned}
$$

The $\theta_{0}$ mode is a new rotated auxiliary field moving with the same velocity $u_{0}=v_{0}=v_{n}$ as the original one. As expected, and as shown below, its dynamics does not enter into the electronic edge correlators.

Eqs. (16]17) imply that the system becomes unstable when $u_{1}<0$, and Eq. (14) indicates that like in the toy model considered at the beginning of this Letter, the instability involves a divergence of the total edge charge $N_{1}+N_{2} \rightarrow \pm \infty$. However, in contrast to the toy model, the instability occurs only once the correlated tunneling strength crosses the following threshold

$$
\lambda>\sqrt{5} \pi \sqrt{v_{c} v_{n}} .
$$

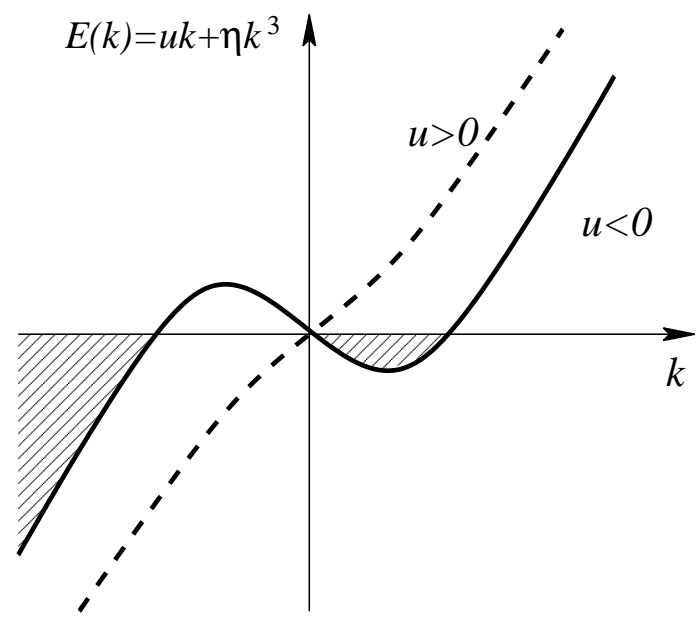

FIG. 1: The dispersion of chiral fermions described by the bosonized action (19). When $u<0$, the fermions occupy the hashed regions and a droplet breaks from the main Fermi sea.

Composite fermions Hartree-Fock calculations of the collective modes of a $\nu=2 / 5$ edge [20] find $v_{c} \sim v_{n} \sim$ $0.05 e^{2} / \epsilon$, where $\epsilon$ is the dielectric constant. Since the correlated tunneling coupling constant is $\lambda \sim e^{2} / \epsilon$, condition (18) may well be satisfied.

Evidently, the appearance of a negative velocity in the action (17) calls for regularization. Higher order nonlinear terms which control the instability appear naturally once corrections to the assumed linear dispersion of the edge composite fermion are taken into account in the CTL action (44). In the course of the various transformations utilized above such terms generate couplings between the modes as well as regularize any divergences. To focus the discussion we ignore the former and consider the effect of a cubic term, $\eta k^{3}$, in the bare edge dispersion, on the unstable mode, whose action is now

$$
\begin{aligned}
S_{u}=\frac{1}{4 \pi} \int d x d \tau & {\left[i \partial_{x} \theta \partial_{\tau} \theta+u\left(\partial_{x} \theta\right)^{2}\right.} \\
+ & \left.\frac{\eta_{2}}{2}\left(\partial_{x}^{2} \theta\right)^{2}+\frac{\eta_{4}}{2}\left(\partial_{x} \theta\right)^{4}\right],
\end{aligned}
$$

where $\eta_{2}$ and $\eta_{4}$ are functions of $\eta$ and the other couplings of our problem. This action my be interpreted as describing chiral fermions with dispersion $E(k)=u k+\eta_{4} k^{3}$ and residual self-interaction $\left(\eta_{2}-\eta_{4}\right)\left(\partial_{x} \rho\right)^{2}$. When the velocity is positive, $u>0$, the nonlinear terms have little effect and the ground state constitutes a Fermi sea that occupies the range $k<0$. However, if $u<0$, the Fermi sea breaks into two distinct components, as illustrated in Fig. 1. and the single chiral channel, which exists before the instability, turns into three. Beside the mode which runs along the shifted edge of the original Fermi sea the instability generates two additional counter-propagating channels on the edges of the detached droplet. Note that 
the instability studied here is different from the one discussed in Ref. 16, where the velocity remains positive for small $k$, and the instability is caused by an interactioninduced negative cubic term in the dispersion.

The effect of the instability on the edge local tunneling density of states (LDOS), as determined by the electronic Green's functions $G_{j}(x=0, \tau)=\left\langle\Psi_{j}(0, \tau) \Psi_{j}^{\dagger}(0,0)\right\rangle$, depends on the nature of the correlations which exist between the electrons in the new droplet. Deferring a discussion of this issue to a future publication [10] we would like to elucidate here the role of correlated tunneling in the LDOS before the instability sets it. Using the fact that the auxiliary field $\phi_{0}$ is completely decoupled from the physical fields, and Eq. (11), we find

$$
\begin{aligned}
G_{j}(x, \tau) & =\frac{\left\langle\xi_{j-1}(x, \tau) \xi_{j-1}^{\dagger}(0,0) e^{i \sqrt{\frac{5}{2}}\left[\varphi_{2}(x, \tau)-\varphi_{2}(0,0)\right]}\right\rangle}{\left\langle e^{\frac{i}{\sqrt{2}}\left[\phi_{0}(x, \tau)-\phi_{0}(0,0)\right]}\right\rangle} \\
& \propto \sum_{\sigma= \pm} e^{i \sigma \frac{\bar{\lambda}}{v_{n}} x} \prod_{j=1}^{2}\left(x+i u_{j} \tau\right)^{-\alpha_{j, \sigma}}
\end{aligned}
$$

where

$$
\alpha_{j, \sigma}=\left[3+(-1)^{j}(2 \cos 2 \gamma+\sqrt{5} \sigma \sin 2 \gamma)\right] / 2 .
$$

Note that the dynamics of the auxiliary field does not appear in the correlators, which reduce in the limit $\lambda \rightarrow 0$ to the known CTL result [18]. Moreover, before the instability $G_{j}(x=0, \tau) \sim \tau^{-3}$; the same power-law predicted by the CTL model for the LDOS of a $\nu=2 / 5$ edge.

We have extended the above analysis to two additional fractions, $\nu=2 / 3$ and $\nu=3 / 7$ [10] and found a similar instability in both cases. Using an analogous action to the one given by Eqs. (48) one obtains that the condition for the instability in the $\nu=2 / 3$ case is $\lambda>\sqrt{3} \pi \sqrt{v_{c} v_{n}}$, where $v_{c}=3(v+g)$ and $v_{n}=v-g$. It turns out that since the charge and neutral modes of the $\nu=2 / 3$ edge propagate in opposite directions, the correlated tunneling processes tend to reduce the tunneling exponent from its CTL value of $\alpha=2$, even before the instability occurs. At filling fraction $\nu=3 / 7$, the edge, which supports one charged mode, with velocity $v_{c}=7(v+2 g)$, and two neutral modes, with velocity $v_{n}=v-g$, reconstructs once $\lambda>\sqrt{7 / 18} \pi \sqrt{v_{c} v_{n}}$. These results suggest a possible breakdown of the naive CTL model for the FQH edge in the entire range $1 / 3<\nu<1$.

We thank Nathan Andrei, Eldad Bettelheim, and Paul Wiegmann for useful discussions. This research was sup- ported by the United States - Israel Binational Science Foundation (grant Nos. 2004162 and 2004128), and by the Israel Science Foundation (ISF) funded by the Israeli Academy of Science and Humanities (grant No. 63/05).

[1] X. G. Wen, Phys. Rev. Lett. 64, 2206 (1990); B. Blok and X. G. Wen, Phys. Rev. B 44, 5708 (1991).

[2] D. Orgad, Phys. Rev. Lett. 79, 475 (1997).

[3] D. Orgad, Phys. Rev. B 75, 035301 (2007).

[4] A. M. Chang, L. N. Pfeiffer, and K. W. West, Phys. Rev. Lett. 77, 2538 (1996).

[5] M. Grayson, D. C. Tsui, L. N. Pfeiffer, K. W. West, and A. M. Chang, Phys. Rev. Lett. 80, 1062 (1998).

[6] A. M. Chang, M. K. Wu, C. C. Chi, L. N. Pfeiffer, and K. W. West, Phys. Rev. Lett. 86, 143 (2001).

[7] M. Hilke, D. C. Tsui, M. Grayson, L. N. Pfeiffer, and K. W. West, Phys. Rev. Lett. 87, 186806 (2001).

[8] For a review of this problem see A. M. Chang, Rev. Mod. Phys. 75, 1449 (2003); L. S. Levitov, A. V. Shytov, and B. I. Halperin, Phys. Rev. B 64, 075322, (2001).

[9] These operators are only approximately fermionic. They obey $\left\{\psi_{i}(\mathbf{r}), \psi_{j}\left(\mathbf{r}^{\prime}\right)\right\}=0$ but $\left\{\psi_{i}^{\dagger}(\mathbf{r}), \psi_{j}\left(\mathbf{r}^{\prime}\right)\right\}=\delta_{i j} \Delta\left(\mathbf{r}, \mathbf{r}^{\prime}\right)$ with $\Delta\left(\mathbf{r}, \mathbf{r}^{\prime}\right) \neq \delta\left(\mathbf{r}-\mathbf{r}^{\prime}\right)$. See Ref. 3 .

[10] D. Orgad and O. Agam, in preparation.

[11] C. de C. Chamon and X. G. Wen, Phys. Rev. B 49, 8227 (1994).

[12] Y. N. Joglekar, H. K. Nguyen, and G. Murthy, Phys. Rev. B 68, 035332 (2003).

[13] E. V. Tsiper and V. J. Goldman, Phys. Rev. B 64, 165311 (2001).

[14] X. Wan, K. Yang, and E. H. Rezayi, Phys. Rev. Lett. 88, 056802 (2002).

[15] X. Wan, E. H. Rezayi, and K. Yang, Phys. Rev. B 68, 125307 (2003).

[16] K. Yang, Phys. Rev. Lett. 91, 036802 (2003).

[17] By their definition $\psi_{i}^{\dagger}(\mathbf{r}) \psi_{j}(\mathbf{r})=\Psi_{i}^{\dagger}(\mathbf{r}) \Psi_{j}(\mathbf{r})$, thus implying a similar equality between the edge projection of the operator products. We assume that the equality holds also for the products of projected operators.

[18] J. D. Naud, L. P. Pryadko and S. L. Sondhi, Nucl. Phys. B 565, 572 (2000).

[19] The original number operator, $N_{i}$ have an integral spectrum. The operators $\mathcal{N}_{i}$ have an integral spectrum or an integral spectrum shifted by $1 / 2$. However, since the tunneling term changes $\mathcal{N}_{0}$ and $\mathcal{N}_{1}$ by \pm 1 , the Hilbert space breaks into sectors which remain disconnected over time.

[20] H. K. Nguyen, Y. N. Joglekar, and G. Murthy, Phys. Rev. B 70, 035324 (2004). 